\title{
Health Policy Brief: i pilastri dell'Engagement in Emofilia
}

\author{
Guendalina Graffigna ${ }^{1}$, Serena Barello ${ }^{1}$, Caterina Bosio ${ }^{1}$, Chiara Biasoli ${ }^{2}$, Andrea Buzzi ${ }^{3}$, Cristina Cassone ${ }^{4}$, Luigi Ambroso ${ }^{4}$, \\ Patrizia Di Gregorio ${ }^{5}$, Lorenzo Farace ${ }^{4}$, Paola Giordano ${ }^{6}$, Renato Marino ${ }^{7}$, Irene Ricca ${ }^{8}$, Angiola Rocino ${ }^{9}$ \\ ${ }^{1}$ Consumer, Food \& Health Engagement Research Center, Department of Psychology, Università Cattolica del Sacro Cuore, Milano - Italy \\ ${ }^{2}$ AICE (Associazione Italiana Centri Emofilia) - Italy \\ ${ }^{3}$ Fondazione Paracelso - Italy \\ ${ }^{4}$ FedEmo (Federazione delle Associazioni Emofilici) - Italy \\ ${ }^{5}$ Servizio di Medicina Trasfusionale Aziendale, Centro Emostasi e Trombosi, Chieti - Italy \\ ${ }^{6}$ Azienda Ospedaliera Universitaria Policlinico di Bari, Centro Emofilia Pediatrico, UOC "B. Trambusti", Bari - Italy \\ ${ }^{7}$ Azienda Ospedaliero Universitaria, Ospedale Policlinico Consorziale, UOSD Emofilia e Trombosi, Bari - Italy \\ ${ }^{8}$ Presidio Ospedale Infantile Regina Margherita/S. Anna, Città della Salute e della Scienza, Centro Emofilia Pediatrico, SSD Trasfusionale \\ Materno-Infantile e Traumatologico, Torino - Italy \\ ${ }^{9}$ Ospedale del Mare, UOC di Ematologia, Centro Emofilia e Trombosi, Napoli - Italy
}

\begin{abstract}
Health Policy Brief: the pillars for Engagement in Hemophilia
About $\mathbf{5 0 0 0}$ people in Italy suffer from hemophilia, the most common coagulation disorder. As for other chronic diseases, even in the case of hemophilia, the engagement of the patient is essential: the patients, in fact, must be empowered and helped to become strong partners of the care team and sensitized with respect to their rights and duties for the successful achievement of the goals set by their healthcare path. Hence the initiative to start a new research-intervention project in the field of hemophilia. The study had different phases of research: a first moment inspired by the principles of narrative medicine, aimed at collecting stories and narratives of patients with hemophilia related to the experience of the disease and therapy and expectations of active involvement in the relationship with the clinician. At the same time, among hematologists and patients has been surveyed the experience of therapeutical relationship and communication, to capture the aspects in which they feel effective and the areas of improvement and unmet needs. Subsequently, a workshop dedicated to patients and hematologists was organized to foster mutual awareness between these two targets and the formation of a better communication and relational skills of clinicians.

The results of the project formed the basis for a policy brief document, aimed at disseminating recommendations to support better relationship and empathic communication between clinicians and patients.
\end{abstract}

Keywords: Engagement, Hemophilia, Patient, Policy brief, Recommendations

\section{Introduzione}

\section{L'emofilia}

L'emofilia è una malattia ereditaria rara dovuta a mutazioni dei geni responsabili della sintesi del fattore VIII (emofilia A) o IX (emofilia B) che determina un'alterazione del normale processo della coagulazione con conseguente aumentato rischio emorragico (1). Nei 2/3 circa dei casi la malattia ha carattere

Received: July 29, 2021

Accepted: December 21, 2021

Published online: January 19, 2022

\section{Corresponding author}

Caterina Bosio

Consumer, Food \& Health Engagement Research Center

Department of Psychology

Università Cattolica del Sacro Cuore

Largo Agostino Gemelli 1

20123 Milano - Italy

caterina.bosio@unicatt.it familiare e viene trasmessa dalla madre portatrice al figlio maschio (eredità diaginica), mentre le figlie femmine possono esserne portatrici e trasmettere, a loro volta, il difetto genetico alla loro prole (2). Nei restanti casi la malattia deriva da una nuova variante del gene F8 o F9 e nella famiglia non sono presenti altri soggetti affetti. II paziente trasmetterà, comunque, la variante genica alle proprie figlie (3). I sintomi caratteristici dell'emofilia A e B sono simili e solo attraverso specifici test di laboratorio è possibile porre la diagnosi differenziale tra i due tipi (3).

Cardine terapeutico di entrambe le malattie è la terapia sostitutiva, attuata "on demand" (ogni volta che compaiono manifestazioni emorragiche) o in regime di profilassi continua con somministrazione di concentrati del fattore carente, secondo uno schema preordinato, allo scopo di prevenire soprattutto gli emartri (manifestazione emorragica tipica dell'emofilia) (3). Il loro ripetersi in singole articolazioni è infatti causa, a lungo andare, dell'instaurarsi di un processo progressivo e incontrovertibile che conduce all'instaurarsi di un quadro da artropatia cronica potenzialmente invalidante con significative ripercussioni sulla qualità della vita e 
sulle possibilità di intrattenere una normale vita sociale (4). L'introduzione della profilassi nella normale pratica clinica ha tuttavia consentito di conseguire una maggiore autonomia $(3,5)$. Nonostante numerose evidenze suggeriscano di iniziarla ancor prima che si manifesti un primo episodio emorragico e sia evidente come questo sarebbe l'approccio terapeutico ideale, esso non viene sempre perseguito, in quanto difficile da attuarsi e perché richiederebbe uno sforzo e una dedizione costanti da parte del paziente e, inizialmente, dei suoi caregiver, da prolungarsi, per giunta, per tutta la vita (6-8).

Per tale motivo, promuovere l'aderenza del paziente alla profilassi risulta determinante per l'efficacia della terapia e per il mantenimento di un buono stato di salute della persona nel lungo periodo. I fattori chiave coinvolti nel processo sono così riassumibili:

- le caratteristiche del paziente: età, le sue percezioni riguardanti la malattia, convinzione dell'efficacia dei farmaci e il proprio livello di autoefficacia percepita, ovvero la propria consapevolezza di essere in grado di dominare la malattia e le sue complicanze e di superare le situazioni di difficoltà che da essa potrebbero derivare (9-13);

- le caratteristiche cliniche: complessità della cura, gravità dei sintomi, dolore acuto e cronico, complicanze potenzialmente invalidanti, comorbilità che potrebbero accentuare il rischio emorragico $(1,14,15)$;

- I'ambiente sociale: supporto strutturato da parte di servizi territoriali, dinamiche familiari e integrazione nel mondo della scuola e del lavoro (1,16-19);

- l'efficacia e l'efficienza dell'assistenza sanitaria ricevuta (informazioni e prescrizioni per la gestione della terapia, supporto per la terapia domiciliare) e la qualità della relazione/comunicazione medico/paziente $(3,19,20)$;

tra i fattori chiave, la qualità della relazione/comunicazione tra paziente e medico rappresenta un elemento di particolare rilevanza sul piano clinico, data la sua plasticità e, quindi, la possibilità di miglioramento adottando opportune azioni di intervento (3,21-25).

Alla luce di queste considerazioni iniziali, le domande a cui il progetto e il presente documento ambiscono a dare risposta sono le seguenti:

- Come sostenere l'aderenza e la migliore qualità di vita della persona con emofilia?

- Come favorire una comunicazione efficace e positiva tra paziente e specialista di riferimento?

- Come sostenere il coinvolgimento attivo del paziente (engagement) nel suo percorso di cura?

\section{Il progetto "Parole in emofilia"}

A partire da queste premesse, nel 2020, ha preso avvio il progetto "Parole in Emofilia", progetto che, ispirandosi ai principi della medicina narrativa, ha voluto dare voce all'esperienza di malattia e di engagement dei pazienti emofilici italiani e dei loro medici di riferimento. Lo studio si è focalizzato, in particolare, sull'identificazione delle modalità relazionali e comunicazionali più idonee e atte a promuovere il coinvolgimento attivo del paziente emofilico nella realtà di cura del nostro Paese.

II progetto, di natura fortemente partecipativa, a cominciare dalla composizione della sua governance, ha visto la combinazione di metodologie qualitative e quantitative, al fine di ottenere una visione ampia e articolata delle storie di comunicazione e partecipazione in emofilia.

Sul piano metodologico lo studio si è articolato su una rilevazione quali-quantitativa coinvolgendo 50 pazienti e 27 specialisti che operano nei Centri di Emofilia distribuiti su tutto il territorio nazionale. Lo studio ha avuto l'obiettivo di dare voce alle priorità e alle aspettative di cura dei pazienti emofilici, esplorando quali siano per loro gli elementi imprescindibili della relazione con il medico di riferimento e i bisogni insoddisfatti, rispetto alle loro esperienze assistenziali, confrontandoli con quelli espressi dal clinico esperto in emofilia. In particolare, l'obiettivo di questa iniziativa è stato creare uno spazio di condivisione di aspettative, desideri e bisogni per migliorare la qualità della relazione tra medici e pazienti e cercare di colmare le lacune ancora esistenti.

Lo studio si è composto di diverse fasi di ricerca: un primo momento qualitativo, ispirato ai principi della medicina narrativa, volto a raccogliere storie e narrazioni di pazienti con emofilia relative all'esperienza della malattia e della terapia e alle aspettative di coinvolgimento attivo nella relazione con il clinico. Allo stesso tempo, è stata indagata, con metodologia quantitativa, attraverso un questionario a risposta chiusa tra ematologi e pazienti, la loro esperienza di relazione terapeutica e di comunicazione, per cogliere gli aspetti su cui si sentono efficaci e le aree di miglioramento e di bisogno insoddisfatto.

I risultati sono stati oggetto di un processo di consenso e integrazione da parte di un panel qualificato di esperti, rappresentati da specialisti nella cura dell'emofilia, e pazienti, e hanno portato alla definizione di alcuni pilastri (raccolti in un documento di Policy Brief) volti al miglioramento della pratica clinica e per la gestione della relazione medicopaziente in questo specifico ambito. È stato infatti organizzato un workshop dedicato ai pazienti e agli ematologi per favorire la conoscenza reciproca tra questi due target e la formazione di migliori capacità comunicative e relazionali dei clinici.

\section{Migliorare l'aderenza alla profilassi attraverso la comu- nicazione medico-paziente in emofilia: la prospettiva del Patient Engagement}

Il modello del Patient Engagement appare di fondamentale importanza ai fini di migliorare l'aderenza alla profilassi, attraverso il miglioramento della comunicazione medico-paziente.

Come noto, una buona comunicazione tra medico e paziente è ampiamente influenzata da fattori soggettivi e contestuali entro cui la relazione si colloca. Ogni individuo, inclusi i pazienti e i loro medici di riferimento, è infatti caratterizzato da aspettative, pensieri e umori differenti, caratteristiche che il clinico e il paziente, ognuno per la propria parte, devono tenere in considerazione al fine di instaurare una relazione duratura che li accompagni nell'intero percorso di vita e di cura della malattia. 
D’altra parte, perché possa instaurarsi un'autentica condizione di medicina partecipativa, appare indispensabile promuovere l'engagement del paziente, ovvero la sua predisposizione psicologica a svolgere un ruolo attivo nel proprio percorso di cura. Il termine engagement deriva dal verbo inglese "to engage" e letteralmente assume il significato di "impegnarsi" o "ingaggiare"; nonostante sia difficile tradurlo in italiano, l'etimo possiede una profonda dimensione relazionale di cui si sostanzia e da cui prende forma. Nella prima conferenza di consenso italiana sul tema della promozione del Patient Engagement, nel 2017, è stata proposta questa definizione: “L'Engagement, nell'ambito clinico assistenziale della cronicità, è un concetto sistemico che identifica e qualifica le possibili modalità di relazione che una persona con una domanda di salute/prevenzione, assistenza e/o cura può intrattenere con la sua condizione clinica, il suo caregiver informale (in particolare la famiglia), il professionista sanitario e il team assistenziale nel suo complesso, il contesto organizzativo, il sistema socio-sanitario $e$ il sistema sociale allargato, durante il proprio percorso clinico assistenziale" (26).

Tra le varie dimensioni coinvolte nel processo, risulta innegabile il ruolo della sfera emotiva, che ne costituisce una leva motivazionale fondamentale. Una persona capace di gestire in maniera efficace le emozioni connesse al proprio stato di salute e di sviluppare un adeguato adattamento alla propria condizione clinica è, infatti, anche in grado di gestire in maniera più efficace il proprio percorso di cura e ha maggiori probabilità di aderire alle prescrizioni del clinico $(1,27)$. Da questo punto di vista emotivo-motivazionale, il Patient Engagement si configura, quindi, come un percorso in cui sono possibili oscillazioni "evolutive e involutive". Ciò è precisamente descritto nel Patient Health Engagement Model (PHE Model) che delinea quattro gradini incrementali di engagement lungo il percorso di adattamento alla propria malattia: Blackout, Allerta, Consapevolezza e Progetto Eudaimonico (28).

La rilevanza di un approccio orientato all'engagement per sostenere l'aderenza del paziente emofilico alla profilassi poggia su alcune evidenze di fondamentale importanza che indicano come questa dimensione giochi un ruolo rilevante nel migliorare gli outcome terapeutici:

- sul piano clinico, documentato sia in riferimento a diverse patologie $(6,29)$ sia in riferimento alle risposte del paziente nel percorso terapeutico (aderenza, fiducia nel medico, adozione di comportamenti preventivi...) (30-32);

- sul piano della qualità di vita (soddisfazione personale, resilienza, auto-realizzazione...) $(3,33)$;

- sul piano dei costi sanitari (bilanciamento tra domanda e offerta di cura, contenimento degli sprechi, utilizzo ottimale delle risorse...) $(32,34,35)$.

\section{I pilastri dell'engagement in emofilia: indicazioni per la relazione terapeutica orientata al coinvolgimento attivo della persona}

In concreto sono identificabili alcuni criteri orientativi per una migliore gestione della relazione terapeutica orientata all'engagement. Il tutto può essere schematizzato come segue:
- accessibilità e trasparenza delle informazioni: favorire l'accesso a informazioni chiare e facilmente accessibili e che considerino le specificità della persona promuovendo, oltre al rapporto diretto con il clinico di riferimento, che rappresenta sempre la fonte di informazione ottimale, in quanto conoscitore delle caratteristiche del singolo paziente, l'accesso ad altre fonti di informazione che siano semplici, chiare e trasparenti (accessibilità a siti web, opuscoli informativi);

- attribuzione di senso: aiutare le persone a integrare il significato dell'esperienza di malattia (emofilia) all'interno di una prospettiva più ampia;

- collaborazione: tra Centro Emofilia e Associazioni dei pazienti al fine di realizzare momenti di confronto e condivisione periodici con lo scopo di favorire la collaborazione tra medico e paziente, principio sul quale si basa la possibilità di assicurare un'ottimale aderenza al regime terapeutico prescritto dal medico del Centro emofilia;

- condivisione delle storie: favorire il confronto e la relazione tra pazienti che condividono simili esperienze di cura e malattia;

- condivisione di obiettivi e scelte: condividere e consensualizzare gli obiettivi e le scelte terapeutiche con il paziente, considerando i suoi valori e le sue aspettative in merito alla qualità di vita attesa;

- formazione del paziente: promuovere un'adeguata formazione del paziente, in modo che sia pienamente consapevole dei propri diritti e doveri e che possa assumere un ruolo di "protagonista attivo" della propria cura;

- immedesimazione-empatia: far emergere e comprendere i sentimenti, le emozioni e le sensazioni con cui ogni persona percepisce la realtà e la malattia;

- inclusione ed equità: impegno a far sì che non vi siano discriminazioni della persona affetta da emofilia anche in termini di rispetto delle specificità di genere, età e caratteristiche culturali, socio-economiche, linguistiche e di culto, garantendo a tutti le medesime possibilità di partecipazione e di engagement nel proprio percorso terapeutico;

- integrazione: promuovere la continuità delle cure, favorendo l'integrazione dei servizi di cura e assistenza a livello territoriale e non solo presso i Centri specialistici per la cura dell'emofilia, in modo da far sì che le cure iniziate/ prescritte dai Centri specialistici possano trovare pronto riscontro presso i Servizi territoriali;

- multidisciplinarità: favorire una presa in carico multidisciplinare della persona con emofilia per rispondere in modo efficace ai bisogni di natura bio-psico-sociale;

- oltre il corpo: considerare le inclinazioni personali, le potenzialità e la vitalità della persona e non solo l'assenza di malattia e il buon funzionamento del corpo come principi guida delle scelte terapeutiche;

- percorso di vita/progettualità: restituire alla persona, anche se affetta da emofilia, la dimensione temporale in cui si collocano la propria esperienza e le sue progettualità future, tenendo conto del vissuto passato e attuale, senza porre limitazioni alcune rispetto alle prospettive future; 
- prevenire è meglio che curare/attivazione delle risorse personali: sensibilizzare e ingaggiare la persona incoraggiandola a tenere conto dell'importanza del proprio ruolo attivo, dalla prevenzione della malattia da cui si è affetti (emofilia) all'aderenza alle terapie di prevenzione delle complicanze;

- responsabilità: valorizzare la capacità della persona con emofilia di agire, fare scelte ed essere responsabile come protagonista attivo delle proprie azioni, anche nel percorso di cura, definendo compiti reciproci di condivisione delle scelte terapeutiche con il medico del Centro emofilia di riferimento all'inizio del trattamento e nel suo prosieguo, mediante un atteggiamento di ascolto e trasparenza bidirezionale, sempre improntato a far sì che la persona affetta da emofilia assuma piena coscienza dei propri limiti e delle proprie potenzialità di assumere un ruolo attivo nella società, grazie agli avanzamenti della terapia oggi disponibili;

- senso del sé: accompagnare il paziente a considerare l'emofilia come uno degli aspetti della propria vita, non l'unico, evitando di identificarsi totalmente con la malattia;

- senso di appartenenza e network: riconoscere il bisogno di appartenenza a un gruppo e di creare e mantenere relazioni significative, da quelle familiari a quelle amicali, a quelle con persone che condividono la stessa esperienza di malattia, favorendo il confronto e la relazione tra persone che condividono simili esperienze e riconoscendo i potenziali benefici che da un tale confronto possono derivare;

- senso di sicurezza e fiducia: consentire a ciascuno di sentirsi al sicuro all'interno di una relazione basata sulla fiducia e di avere punti di riferimento stabili lungo il percorso terapeutico con cui confrontarsi costantemente;

- unicità: riconoscere l'unicità della persona, pur nel suo appartenere all'insieme delle persone o dei pazienti affetti da emofilia;

- valorizzazione dei propri luoghi di vita: completamento della presa in carico di ogni persona/paziente affetto da emofilia con restituzione, quando possibile, al proprio ambiente di vita, eventualmente sostenuta da adeguati supporti all'inserimento nella vita sociale (valorizzando in particolare il ruolo del caregiver e il supporto delle associazioni dei pazienti, degli operatori sociali che agiscono a livello territoriale, degli operatori scolastici, ecc.).

\section{Conclusioni e possibili sviluppi futuri}

I pilastri descritti scaturiscono da una ricerca narrativa volta a dare voce ai pazienti e che rappresenta solo la prima fase di un progetto di più ampio respiro, che coinvolgerà pazienti e medici che operano nei Centri Emofilia di loro riferimento. Quanto acquisito finora ha già consentito di definire una serie di priorità condivise, volte a favorire l'engagement della persona con emofilia in un'efficace relazione di cura con il proprio medico. Tali pilastri trovano piena conferma nella letteratura scientifica sul "Patient Engagement" e sulla promozione del coinvolgimento del paziente con una patologia cronica, come l'emofilia, in un percorso di piena condivisione delle scelte terapeutiche che lo coinvolgano direttamente.
Nello specifico, nel considerare come sviluppare al meglio un sistema efficace che abbini qualità dell'assistenza e valore per i cittadini e che sia in grado di soddisfare la domanda di assistenza, il ruolo svolto dai pazienti è diventato sempre più importante, tanto da conferire loro un ruolo da protagonisti che, oggi, d'altra parte, si pone come elemento prioritario, per le politiche sanitarie, sia a livello etico che a livello pragmatico. La letteratura scientifica internazionale, infatti, evidenzia come la promozione della partecipazione attiva dei pazienti ("Patient Engagement") produca risultati considerevoli, tra i quali:

- facilitazione nella gestione della persona per migliorare gli outcome clinici. Dalla letteratura si evidenzia, infatti, il ruolo del Patient Engagement nel migliorare la condizione clinica trasversalmente alle diverse patologie $(29,36-$ 38). Per esempio, alcuni studi hanno dimostrato come i pazienti più attivamente coinvolti nei loro piani di cura fossero più inclini a fidarsi dei propri clinici di riferimento (30) e più aderenti alle prescrizioni terapeutiche (1). Hibbard et al. hanno anche evidenziato una diminuzione di eventi clinici e di ri-ospedalizzazioni. II Patient Engagement sembra, inoltre, contribuire al perseguimento di piani di vita sostenibili e a evitare di assumere condotte non salutari (39-41);

- miglioramento della soddisfazione e della qualità di vita della persona: il Patient Engagement emerge dai contributi scientifici come un elemento chiave per favorire la crescita personale e la partecipazione delle persone nel percorso di cura, ma anche nella comunità di riferimento, promuovendo la soddisfazione della persona e favorendo le sue opportunità di azione e di espressione $(3,42)$. Inoltre, il Patient Engagement può contribuire al miglioramento della qualità di vita dei pazienti con l'obiettivo di aumentare il benessere e di generare forza e resilienza negli individui che devono affrontare eventi acuti di malattia (43);

- riduzione dei costi sanitari: il dibattito scientifico sottolinea come il Patient Engagement, in termini di maggiore sensibilizzazione dei pazienti, di acquisizione di conoscenze e di competenze e di maggiore empowerment delle persone con malattia cronica, risulti cruciale nel contribuire a un adeguato bilanciamento tra l'aumento della richiesta di cura e la scarsità delle risorse economiche sanitarie disponibili (34), riducendo i costi diretti e ri-orientando le risorse esistenti in una gestione più efficace e sostenibile, volta a evitare sprechi economici senza comportare una riduzione della qualità dell'assistenza prestata ai pazienti (44). Mentre ci muoviamo verso il futuro in medicina e nel trattamento dell'emofilia, porre il paziente al "centro" dei paradigmi di cura diventa, infatti, inevitabile per rendere l'assistenza sanitaria più significativa, di impatto e sostenibile a livello economico, sociale e psicologico (35).

D’altro canto, non esiste una regola universale che permetta di instaurare una buona relazione tra medico e paziente perché tale legame ha una forte variabilità a causa dei fattori soggettivi e contestuali che ne sono alla base. Ogni persona è caratterizzata da aspettative, pensieri e umori 
differenti e ognuna di queste caratteristiche deve essere tenuta in considerazione dal clinico al fine di instaurare una relazione duratura, che accompagni la persona con emofilia durante il suo percorso di cura. La fiducia può essere considerata il principale collante di questo legame (45). Sul significato di tale termine, collocato all'interno della relazione-medico paziente, si sono espressi vari autori non arrivando mai a un significato univoco definitivo, a causa della natura molto labile e delle molteplici sfaccettature di senso che esso trascina con sé. Per esempio, Thom e Campbell (46) hanno identificato alcuni fattori fondamentali su cui si basa la costruzione di una relazione di engagement e di fiducia tra paziente e operatore sanitario: condividere i problemi, comprendere l'esperienza individuale del paziente, esprimere comprensione ed empatia, fornire un trattamento adeguato ed efficace, comunicare in modo chiaro e completo, costruire rapporti di alleanza e partnership, condividere le scelte, dimostrare onestà intellettuale e rispettare la persona. Pertanto, un medico esperto che si concentri solo sulle condizioni cliniche dei pazienti non sarebbe in grado di costruire un rapporto di fiducia reciproca. Al di là dell'attribuzione di significato che si conferisce al termine "fiducia", lo sforzo per il raggiungimento non è dettato soltanto da principi etici, ma si concretizza in benefici di ineluttabile importanza sul piano individuale e sociosanitario.

Condizioni necessarie affinché possa essere perseguito l'obiettivo di una Medicina Partecipativa sono la promozione e la sensibilizzazione dei cittadini/pazienti all'engagement, vale a dire la predisposizione psicologica a giocare un ruolo attivo e partecipe nel proprio percorso di cura.

Altri autori fanno riferire il concetto di Patient Engagement alle abilità acquisite dalla persona di adottare uno stile di vita sano (47) oppure, ancora in riferimento alle conoscenze e all'autoefficacia percepita, alla coscienza e alla capacità di assumere un ruolo attivo nella gestione della propria salute fisica e psichica (32). Al di là delle numerose definizioni, è innegabile che nel processo di engagement sia sempre coinvolta la sfera emotiva, che ne costituisce una leva motivazionale fondamentale. Infatti, un individuo capace di gestire, in maniera efficace, le emozioni connesse alla propria malattia e di sviluppare un adeguato adattamento alla propria condizione di salute è anche un individuo più efficace nella gestione del suo percorso sanitario e ha maggiori probabilità di aderire alle prescrizioni mediche (48). Inoltre, questa stessa leva è stata dimostrata essere in relazione con una maggiore consapevolezza del paziente nell'ascoltare il proprio corpo e nel riconoscere i propri sintomi, diventando, quindi, capace di consultare tempestivamente i professionisti sanitari. Tuttavia, il processo di engagement ottimale non è un processo immediato, non si tratta di un on/off, ma si realizza in maniera graduale. II Patient Health Engagement Model (PHE Model) delinea, infatti, i quattro scalini di engagement che segnano la traiettoria su cui muoversi lungo il percorso di adattamento alla propria malattia (49).

Alla luce di queste considerazioni, questo documento sottolinea una serie di opportunità per orientare la relazione medico-paziente nell'ambito delle malattie emorragiche congenite e dell'emofilia, in particolare, nella prospettiva del Patient Engagement e della promozione e della facilitazione dell'aderenza terapeutica. Lo specialista del Centro Emofilia, in relazione agli sviluppi del percorso clinico e della comunicazione che lo sostiene, potrà attingere ai suggerimenti più opportuni per un miglioramento della relazione.

Al di là di questo impiego immediato, gli esperti del settore concordano sull'opportunità di proseguire lungo il percorso qui accennato nella direzione di una quantificazione dell'importanza di ciascun pilastro, che consenta di favorire un orientamento all'engagement della persona affetta da emofilia. Essi sottolineano, inoltre, l'opportunità di promuovere pratiche di riconoscimento di valore delle organizzazioni virtuose che aderiscano ai principi di engagement identificati in questo documento riconosciuti e attesi sia dai pazienti che dai loro medici di riferimento, promuovendoli. Questo non in ottica di una sanzione giudicatoria ma, al contrario, come via maestra da percorrere per promuovere partecipazione e collaborazione tra i diversi stakeholder nella direzione di un reale cambiamento delle prassi sanitarie con l'obiettivo di raggiungere il traguardo di una medicina realmente partecipativa.

\section{Acknowledgements}

EngageMinds HUB desidera ringraziare la Società Scientifica (AICE - Associazione Italiana Centri Emofilia) e le Associazioni Pazienti (FedEmo - Federazione delle Associazioni Emofilici e Fondazione Paracelso) che hanno preso parte alla Cabina di Regia del progetto.

\section{Disclosures}

Conflict of interest: The Authors declare no conflict of interest. Financial support: This study was funded by an unconditional grant form Kedrion Biopharma.

\section{Bibliografia}

1. Hibbard JH, Greene J, Tusler M. Improving the outcomes of disease management by tailoring care to the patient's level of activation. Am J Manag Care. 2009;15(6):353-360. CrossRef PubMed

2. Acquila M, Bicocchi P, Frusconi S, Lombardi AM. Linee guida per la diagnosi molecolare di Emofilia A. Online

3. Thornburg CD, Carpenter S, Zappa S, et al. Modern haemophilia treatment: medical improvements and quality of life. Haemophilia. 2013;4(4):91.

4. Schrijvers L, Beijlevelt-Van der Zande M, Peters M, et al. Achieving self-management of prophylactic treatment in adolescents: the case of haemophilia. Patient Educ Couns. 2016;99(7):11791183. CrossRef PubMed

5. Rosendaal FR, Smit C, Varekamp I, et al. Modern haemophilia treatment: medical improvements and quality of life. J Intern Med. 1990;228(6):633-640. CrossRef PubMed

6. Alexander JA, Hearld LR, Mittler JN, Harvey J. Patient-physician role relationships and patient activation among individuals with chronic illness. Health Serv Res. 2012;47(3 Pt 1):12011223. CrossRef PubMed

7. McLaughlin JM, Witkop ML, Lambing A, Anderson TL, Munn J, Tortella B. Better adherence to prescribed treatment regimen is related to less chronic pain among adolescents and young adults with moderate or severe haemophilia. Haemophilia. 2014;20(4):506-512. CrossRef PubMed 
8. Poti S, Palareti L, Cassis FRMY, Brondi S. Health care professionals dealing with hemophilia: insights from the international qualitative study of the HERO initiative. J Multidiscip Healthc. 2019;12:361-375. CrossRef PubMed

9. Berntorp $E$, Boulyjenkov V, Brettler $D$, et al. Modern treatment of haemophilia. Bull World Health Organ. 1995;73(5):691-701. PubMed

10. Horne R, Parham R, Driscoll R, Robinson A. Patients' attitudes to medicines and adherence to maintenance treatment in inflammatory bowel disease. Inflamm Bowel Dis. 2009;15(6):837-844. CrossRef PubMed

11. Lamiani G, Strada I, Mancuso ME, Coppola A, Vegni E, Moja EA; Pro-Adherence Study Group. Factors influencing illness representations and perceived adherence in haemophilic patients: a pilot study. Haemophilia. 2015;21(5):598-604. CrossRef PubMed

12. Watson D, Clark LA, Tellegen A, et al. The behaviour and perception of illness: modulating variables of adherence in patients with haemophilia. Haemophilia. 2017;18(6):577-583.

13. Tran DQ, Barry V, Antun A, Ribeiro M, Stein S, Kempton CL. Physician trust and depression influence adherence to factor replacement: a single-centre cross-sectional study. Haemophilia. 2017;23(1):98-104. CrossRef PubMed

14. Breakey VR, Blanchette VS, Bolton-Maggs PHB. Towards comprehensive care in transition for young people with haemophilia. Haemophilia. 2010;16(6):848-857. CrossRef PubMed

15. Hacker MR, Geraghty S, Manco-Johnson M. Barriers to compliance with prophylaxis therapy in haemophilia. Haemophilia. 2001;7(4):392-396. PubMed

16. Geraghty S, Dunkley T, Harrington C, Lindvall K, Maahs J, Sek J. Practice patterns in haemophilia A therapy - global progress towards optimal care. Haemophilia. 2006;12(1):75-81. CrossRef PubMed

17. Iannone $M$, Pennick $L$, Tom $A$, et al. Prevalence of depression in adults with haemophilia. Haemophilia. 2012;18(6):868-874. CrossRef PubMed

18. Miller TA. Health literacy and adherence to medical treatment in chronic and acute illness: A meta-analysis. Patient Educ Couns. 2016;99(7):1079-1086. CrossRef PubMed

19. von der Lippe C, Frich JC, Harris A, Solbrække KN. "It was a lot tougher than I thought it would be". A qualitative study on the changing nature of being a hemophilia carrier. J Genet Couns. 2017;26(6):1324-1332. CrossRef PubMed

20. Fischer K, van der Bom JG, Molho P, et al. Prophylactic versus on-demand treatment strategies for severe haemophilia: a comparison of costs and long-term outcome. Haemophilia. 2002;8(6):745-752. CrossRef PubMed

21. Beard JR, Officer A, de Carvalho IA, et al. The World report on ageing and health: a policy framework for healthy ageing. Lancet. 2016;387(10033):2145-2154. CrossRef PubMed

22. Krishnan S, Vietri J, Furlan R, Duncan N. Adherence to prophylaxis is associated with better outcomes in moderate and severe haemophilia: results of a patient survey. Haemophilia. 2015;21(1):64-70. CrossRef PubMed

23. Ono O, Suzuki Y, Yosikawa $\mathrm{K}$, et al; Planning Committee, Japanese Nurse Group for Research of Hemophilia Care. Assessment of haemophilia treatment practice pattern in Japan. Haemophilia. 2009;15(5):1032-1038. CrossRef PubMed

24. Petrini P. Identifying and overcoming barriers to prophylaxis in the management of haemophilia. Haemophilia. 2007;13(s2) (suppl 2):16-22. CrossRef PubMed

25. Zappa S, McDaniel M, Marandola J, Allen G. Treatment trends for haemophilia A and haemophilia B in the United States: results from the 2010 practice patterns survey. Haemophilia. 2012;18(3):e140-e153. CrossRef PubMed
26. Graffigna G, Barello S, Riva G, et al; CCIPE Working Group. Promozione del patient engagement in ambito clinico-Assistenziale per le malattie croniche: raccomandazioni dalla prima conferenza di consenso italiana. Recenti Prog Med. 2017;108(11): 455-475. CrossRef PubMed

27. Graffigna $G$, Barello $S$, Bonanomi A. The role of Patient Health Engagement Model (PHE-model) in affecting patient activation and medication adherence: A structural equation model. PLOS One. 2017;12(6):e0179865. CrossRef PubMed

28. Graffigna G, Barello S. Spotlight on the Patient Health Engagement model (PHE model): a psychosocial theory to understand people's meaningful engagement in their own health care. $\mathrm{Pa}$ tient Prefer Adherence. 2018;12:1261-1271. CrossRef PubMed

29. Ng M, Fleming $T$, Robinson $M$, et al. Global, regional, and national prevalence of overweight and obesity in children and adults during 1980-2013: a systematic analysis for the Global Burden of Disease Study 2013. Lancet. 2014;384(9945): 766-781. CrossRef PubMed

30. Maiman LA, Becker MH. The Health Belief Model: Origins and Correlates in Psychological Theory. Health Educ Monogr. 1974;2(4):336-353. CrossRef

31. Frenk J, Chen L, Bhutta ZA, et al. Health professionals for a new century: transforming education to strengthen health systems in an interdependent world. Lancet. 2010;376(9756):19231958. CrossRef PubMed

32. Hibbard JH, Greene J. What the evidence shows about patient activation: better health outcomes and care experiences; fewer data on costs. Health Aff (Millwood). 2013;32(2):207-214. CrossRef PubMed

33. Webb SM, Martínez-Momblán MA, Resmini E, Valassi E, Santos A. Quality of life in patients with pituitary tumors. Curr Opin Endocr Metab Res. 2018;1:67-73. CrossRef

34. Barello S, Graffigna G, Pitacco G, Mislej M, Cortale M, Provenzi L. An Educational Intervention to Train Professional Nurses in Promoting Patient Engagement: A Pilot Feasibility Study. Front Psychol. 2017;7:2020. CrossRef PubMed

35. Johansen NJ, Saunders CM. Value-Based Care in the Worldwide Battle Against Cancer. Cureus. 2017;9(2):e1039. CrossRef PubMed

36. Estes A, Munson J, Dawson G, Koehler E, Zhou X-H, Abbott R. Parenting stress and psychological functioning among mothers of preschool children with autism and developmental delay. Autism. 2009;13(4):375-387. CrossRef PubMed

37. Dewey KG, Begum K. Long-term consequences of stunting in early life. Matern Child Nutr. 2011;7(suppl 3):5-18. CrossRef PubMed

38. van Deursen AJAM. Internet skill-related problems in accessing online health information. Int J Med Inform. 2012;81(1):61-72. CrossRef PubMed

39. Epping-Jordan JE, Pruitt SD, Bengoa R, Wagner EH. Improving the quality of health care for chronic conditions. Qual Saf Health Care. 2004;13(4):299-305. CrossRef PubMed

40. Huttner A, Harbarth S, Carlet J, et al. Antimicrobial resistance: a global view from the 2013 World Healthcare-Associated Infections Forum. Antimicrob Resist Infect Control. 2013;2(1):31. CrossRef PubMed

41. Ginsburg L, Berta W, Baumbusch J, et al. Measuring work engagement, psychological empowerment, and organizational citizenship behavior among health care aides. Gerontologist. 2016;56(2):e1-e11. CrossRef PubMed

42. Brom L, Hopmans W, Pasman HR, Timmermans DR, Widdershoven GA, Onwuteaka-Philipsen BD. Congruence between patients' preferred and perceived participation in medical decision-making: a review of the literature. BMC Med Inform Decis Mak. 2014 Apr 3;14:25. CrossRef PubMed 
43. Bediako SM, Lanzkron S, Diener-West M, Onojobi G, Beach MC, Haywood C Jr. The measure of sickle cell stigma: initial findings from the improving patient outcomes through respect and trust study. J Health Psychol. 2016;21(5):808-820. CrossRef PubMed

44. Hibbard JH, Greene J, Shi Y, Mittler J, Scanlon D. Taking the long view: how well do patient activation scores predict outcomes four years later?. Medical Care Research and Review. 2015; 72(3): 324-337. CrossRef PubMed

45. Thornburg CD, Duncan NA. Treatment adherence in hemophilia. Patient Prefer Adherence. 2017;11:1677-1686. CrossRef PubMed
46. Thom DH, Campbell B. Patient-physician trust: an exploratory study. J Fam Pract. 1997;44(2):169-176. PubMed

47. Hibbard JH, Stockard J, Mahoney ER, Tusler M. Development of the Patient Activation Measure (PAM): Conceptualizing and measuring activation in patients and consumers. Health Serv Res. 2004;39(4p1):1005-1026. CrossRef PubMed

48. Gruman J, Rovner MH, French ME, et al. From patient education to patient engagement: implications for the field of patient education. Patient Educ Couns. 2010;78(3):350-356. CrossRef PubMed

49. Graffigna, G. SB. Engagement. Un nuovo modello di partecipazione in sanità. Roma: II Pensiero Scientifico; 2017. 